\title{
Characterization of Phonon Vibrations of Silica Bilayer Films
}

\author{
Nina F. Richter, ${ }^{\dagger}$ Felix E. Feiten, ${ }^{\ddagger}$ Jagriti Pal, $^{\ddagger}$ Agata Plucienik, ${ }^{\ddagger}$ Emre Emmez, ${ }^{\ddagger}$ Shamil Shaikhutdinov, ${ }^{\ddagger}$

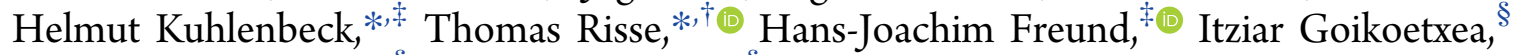 \\ Radosław Włodarczyk, ${ }^{\S}$ and Joachim Sauer ${ }^{\S}$ \\ ${ }^{\dagger}$ Institut für Chemie und Biochemie, Freie Universität Berlin, Takustr. 3, 14195 Berlin, Germany \\ ${ }^{\ddagger}$ Fritz-Haber-Institut der Max-Planck-Gesellschaft, Faradayweg 4-6, 14195 Berlin, Germany \\ ${ }^{\S}$ Institut für Chemie, Humboldt Universität zu Berlin, Unter den Linden 6, 10099 Berlin, Germany
}

Supporting Information

\begin{abstract}
The vibrational properties of well-defined, two-dimensional silica films grown on $\mathrm{Ru}(0001)$ are characterized by highresolution electron energy loss spectroscopy (HREELS). It is an interesting model system because it can adopt both crystalline and vitreous states. A transformation between these states induced by thermal annealing does hardly change the vibrational spectrum despite the redistribution of ring sizes. This holds good for the two intense phonon modes as well as for a variety of weaker modes observed by HREELS. The HREELS spectra allow the characterization of the structural arrangement of the oxygen atoms on the $\mathrm{Ru}(0001)$ surface underneath the silica bilayer. The density of oxygen at the interface can be controlled by the oxygen partial pressure during annealing, resulting

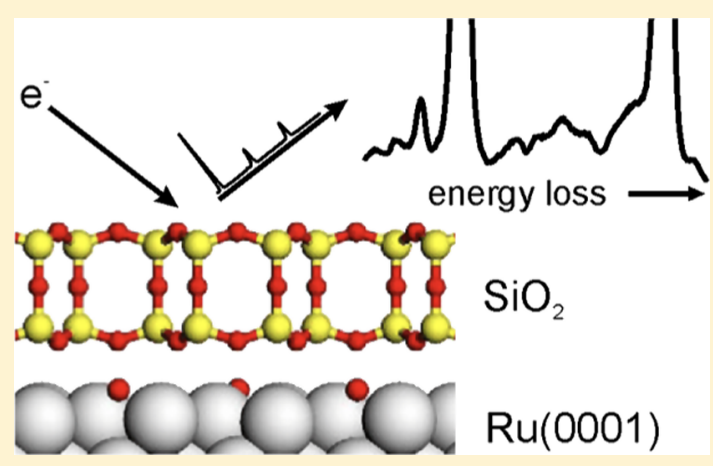
in a characteristic change of the corresponding signals, which can be assigned to different oxygen structures based on density functional theory calculations. By comparison with quantum mechanical calculations and spectroscopic results from the literature, we assign most of the remaining weak signals observed here to the dipole-inactive modes of the bilayer film, structural imperfections such as patches of monolayer structure, and additional silica particles on top of the bilayer film.
\end{abstract}

\section{INTRODUCTION}

Silicon dioxide has a great variety of technical applications, which renders atomistic understanding of its properties important. Because of the very rich structural diversity found in silica materials, the use of appropriate model systems offers interesting opportunities to elucidate various properties in detail. Ultrathin silicon dioxide films supported on metal substrates were shown to be interesting model systems, which have gained considerable attention in the last couple of years. ${ }^{1-9}$ The ultrathin silica films are built from corner sharing $\left[\mathrm{SiO}_{4}\right]$ tetrahedra. The first-ordered silica film was a monolayer structure grown on $\mathrm{Mo}(112)$, which consists of a network of hexagonal rings with a nominal composition of $\mathrm{SiO}_{2.5} \cdot{ }^{2,6,10,11}$ Whereas three of the corners form the hexagonal arrangement in the surface layer, the film is covalently attached to the metal support through the fourth corner. ${ }^{12}$ On noble metals such as $\mathrm{Pt}(111), \operatorname{Pd}(111)$, and $\mathrm{Ru}(0001)$, it is possible to form covalently saturated bilayer structures, which interact only weakly with the metal support. ${ }^{12-16}$ One interesting aspect of these bilayers is the ability to deviate from the crystalline arrangement of hexagonal rings to form vitreous films composed of an irregular arrangement of four- to ninemembered rings. ${ }^{7,17,18}$ In a recent study, vibrational spectroscopy, namely, Raman spectroscopy, on two-dimensional silica materials suggested that low-energy phonons might allow to discriminate between the crystalline and vitreous phases of the material. ${ }^{19}$ To elucidate this point for metal-supported films, high-resolution electron energy loss spectroscopy (HREELS) is a suitable technique as it allows to take surface-sensitive vibrational spectra without the necessity to obey the dipole selection rules.

The weak interaction between the film and the $\mathrm{Ru}(0001)$ surface results in a significant distance between the film and the substrate, which was calculated to range between 2.75 and 3.85 $\AA$, depending on the oxygen coverage remaining on the $\mathrm{Ru}(0001)$ surface. $^{20}$ It was shown that the intercalation of small gas molecules such as $\mathrm{CO}$ and $\mathrm{D}_{2}$ as well as the intercalation of small metal atoms such as $\mathrm{Pd}$ in the interface between silica bilayer films and $\mathrm{Ru}(0001)$ are possible. ${ }^{21,22}$ The oxygen atoms can be reacted with hydrogen to form water, which is interesting because of the confined space in which the reaction takes place. ${ }^{23}$ Oxygen chemisorbed on $\mathrm{Ru}(0001)$ has been characterized in detail by quantum mechanical calculations and experiments previously, however, the expected stretching frequencies below about $600 \mathrm{~cm}^{-1}$

Received: October 28, 2018

Revised: February 12, 2019

Published: March 7, 2019 


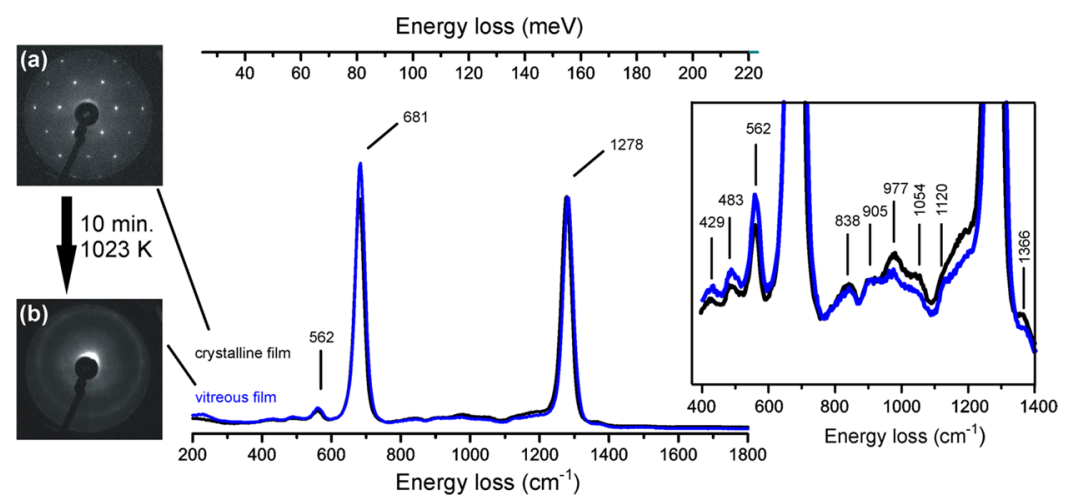

Figure 1. HREELS spectra of the phonon region and LEED pattern of crystalline [black trace, LEED pattern (a)] and vitreous [blue trace, LEED pattern (b)] silica bilayer films supported on $\mathrm{Ru}(0001)$. Spectra are normalized to the high-energy phonon at $1278 \mathrm{~cm}^{-1}$.

render characterization using IR spectroscopy challenging. $^{24-30}$

Herein, we present HREELS investigations of silica bilayer films on $\mathrm{Ru}(0001)$ to characterize the vibrational modes of silica films on $\mathrm{Ru}(0001)$ in the mid-infrared (IR) range, which complements previous IR spectroscopic as well as recent helium atom scattering experiments. ${ }^{13,31}$ In particular, we aim at elucidating the question if vibrational spectroscopy is capable of discriminating between the crystalline and vitreous forms of the bilayer. Apart from the two main phonon peaks characterized in the literature by IR spectroscopy, the films show a variety of additional bands whose speciation and composition depend on the details of the preparation conditions. We assign these bands to the vibrational modes of the bilayer film, structural defects, oxygen atoms residing at the $\mathrm{Ru}$ metal surface beneath the silica film.

\section{EXPERIMENTAL DETAILS}

Experiments were performed in an ultrahigh vacuum (UHV) system equipped with low-energy electron diffraction (LEED), a mass spectrometer (QMS, Hiden 201), an ion-gun, and an EFM 3 evaporator (Omicron), to allow the preparation of the $\mathrm{SiO}_{2}$ bilayer on $\mathrm{Ru}(0001)$. In addition, the system is equipped with a high-resolution electron energy loss spectrometer (Delta 0.5 , VSI). The fixed angle of the electron source in combination with an adjustable angle of the analyzer allow HREELS measurements in specular and off-specular geometries.

A $\mathrm{Ru}(0001)$ crystal (99.99\%, MaTeck) was mounted on a homemade sample holder with a heating filament mounted on the backside of the crystal, which allows for electron beam heating of the sample. The temperature was controlled by a type $\mathrm{K}$ thermocouple spot-welded to the edge of the crystal. The thermocouple was calibrated at room temperature (RT) and liquid nitrogen temperature, resulting in an error at a low temperature of $1-2 \mathrm{~K}$.

The $\mathrm{Ru}(0001)$ crystal was cleaned by repeated cycles of argon-ion sputtering and vacuum annealing to $\sim 1300 \mathrm{~K}$. The $\mathrm{SiO}_{2}$ bilayer preparation consisted of three steps. At first, the metal support was covered with an oxygen layer by annealing to $1200 \mathrm{~K}$ for $10 \mathrm{~min}$ in an oxygen atmosphere of $3 \times 10^{-6}$ mbar. Second, an amount of Si required to form the bilayer (in short 2 MLE) was evaporated at RT from a Si rod onto the $3 \mathrm{O}(2 \times 2)-\mathrm{Ru}(0001)$ surface in an oxygen atmosphere of $2 \times$ $10^{-7}$ mbar. Finally, the sample was annealed to $\sim 1200 \mathrm{~K}$ in an oxygen atmosphere of $2 \times 10^{-6}$ mbar for $10 \mathrm{~min}$, resulting in an ordered $\mathrm{SiO}_{2}$ bilayer film as verified by LEED and HREELS.

All HREELS spectra were measured at RT with an incident beam energy of $6 \mathrm{eV}$. The spectra recorded for specular geometry were measured with an analyzer angle of $67^{\circ}$ relative to the surface normal and a full width at half-maximum resolution of $15-22 \mathrm{~cm}^{-1}(1.8-2.7 \mathrm{meV})$ of the elastic peak. In addition to the specular geometry, some spectra were measured in the $6^{\circ}$ off-specular geometry.

\section{COMPUTATIONAL DETAILS}

The calculations were carried out using Vienna $\mathrm{Ab}$ initio Simulation Package (VASP) ${ }^{32,33}$ along with the Perdew, Burke and Ernzerhof $(\mathrm{PBE})^{34,35}$ exchange-correlation functional. The electron-ion interactions are described by the projector augmented wave method, originally developed by Blöchl ${ }^{36}$ and adapted by Kresse and Joubert. ${ }^{37}$ Only the valence electrons are explicitly considered. Semiempirical dispersion correction is added to include the dispersion forces $(\mathrm{PBE}+\mathrm{D}) .^{38-40}$

The $\mathrm{Ru}(0001)$ slabs were modeled using an orthorhombic $(2 \times 2)$ supercell, $a_{0}=5.396 \AA$ and $b_{0}=9.346 \AA$, containing five $\mathrm{Ru}$ layers; two of them were fixed to their bulk positions and three were relaxed. We used $400 \mathrm{eV}$ cutoff for the planewave basis set. The integrations in the Brillouin zone were performed using a $(8 \times 4 \times 1)$ Monkhorst-Pack grid. ${ }^{41}$

Harmonic vibrational frequencies are calculated using a central finite difference method with $0.02 \AA$ displacements of the atoms in each Cartesian direction. To compensate for systematic errors of density functional theory (DFT), the vibrational frequencies are scaled by an empirical factor of 1.0341 derived from a comparison between experimental ${ }^{42,43}$ and calculated frequencies for $\alpha$-quartz (see the Supporting Information for details).

\section{RESULTS AND DISCUSSION}

Silica bilayers on $\mathrm{Ru}(0001)$ were shown to grow in crystalline and vitreous forms, which can coexist and interconvert depending on the preparation conditions. ${ }^{13}$ In the vitreous form of the film, the regular arrangement of hexagonal rings on the surface plane is replaced by a network of four- to ninemembered rings, however, the registry between the two leaflets remains, which keeps the system covalently saturated. ${ }^{12}$ Two characteristic phonon signals were observed by infrared reflection absorption spectroscopy (IRAS), and DFT calculations were used to show that their positions are in line with the structure of the crystalline form. ${ }^{13}$ The $\mathrm{SiO}_{2}$ films are, 
Table 1. HREELS Signals Observed for the Films Presented in Figures 1-4

\begin{tabular}{|c|c|c|c|}
\hline signal & assignment & signal & assignment \\
\hline bilayer & & monolayer & \\
\hline $1366 \mathrm{~cm}^{-1}(169.4 \mathrm{meV})$ & overtone of mode @681 cm ${ }^{-1}$ [this work] & $1134 \mathrm{~cm}^{-1}(140.6 \mathrm{meV})$ & dipole allowed mode ${ }^{12}$ \\
\hline $1278 \mathrm{~cm}^{-1}(158.5 \mathrm{meV})$ & dipole allowed mode ${ }^{13}$ & $1060 \mathrm{~cm}^{-1}(131.1 \mathrm{meV})$ & dipole allowed mode ${ }^{12}$ \\
\hline \multirow[t]{2}{*}{$1054 \mathrm{~cm}^{-1}(130.7 \mathrm{meV})$} & dipole forbidden mode; Raman-active for a Kagome lattice ${ }^{19}$ & $784 \mathrm{~cm}^{-1}(97.2 \mathrm{meV})$ & dipole allowed mode ${ }^{12}$ \\
\hline & & $677 \mathrm{~cm}^{-1}(84 \mathrm{meV})$ & dipole allowed mode ${ }^{12}$ \\
\hline $905 \mathrm{~cm}^{-1}(112.2 \mathrm{meV})$ & & $540 \mathrm{~cm}^{-1}(67 \mathrm{meV})$ & [this work $]$ \\
\hline $838 \mathrm{~cm}^{-1}(103.9 \mathrm{meV})$ & & $463 \mathrm{~cm}^{-1}(57.4 \mathrm{meV})$ & $\begin{array}{l}\text { dipole allowed mode [this } \\
\text { work] }\end{array}$ \\
\hline $681 \mathrm{~cm}^{-1}(84.4 \mathrm{meV})$ & dipole allowed mode ${ }^{13}$ & $308 \mathrm{~cm}^{-1}(30.2 \mathrm{meV})$ & $\begin{array}{l}\text { dipole allowed mode [this } \\
\text { work] }\end{array}$ \\
\hline $483 \mathrm{~cm}^{-1}(59.9 \mathrm{meV})$ & $\begin{array}{l}\text { dipole forbidden mode; mode at } 454 \text { and } 500 \mathrm{~cm}^{-1} \text { observed } \\
\text { in Raman spectra }{ }^{19}\end{array}$ & & \\
\hline $429 \mathrm{~cm}^{-1}(53.2 \mathrm{meV})$ & $\begin{array}{l}\text { dipole forbidden mode; mode at } 409 \mathrm{~cm}^{-1} \text { observed in Raman } \\
\text { spectra }\end{array}$ & & \\
\hline 3D particles on top of silica bilayer & & $\begin{array}{l}\text { modes due to oxygen atom } \\
\text { system }\end{array}$ & for the $\mathrm{SiO}_{2} / \mathrm{O} / \mathrm{Ru}(0001)$ \\
\hline $\begin{array}{l}1260 \mathrm{~cm}^{-1}(156.3 \mathrm{meV}) \text { to } 1150 \\
\mathrm{~cm}^{-1}(142.9 \mathrm{meV})\end{array}$ & dipole allowed mode $\mathrm{e}^{3,4,44-50}$ & $624 \mathrm{~cm}^{-1}(77.4 \mathrm{meV})$ & $\begin{array}{l}\text { dipole allowed mode [this } \\
\text { work] }\end{array}$ \\
\hline \multirow[t]{2}{*}{$977 \mathrm{~cm}^{-1}(121.1 \mathrm{meV})$} & dipole allowed mode 44,50 & $564 \mathrm{~cm}^{-1}(70 \mathrm{meV})$ & $\begin{array}{l}\text { dipole allowed mode [this } \\
\text { work] }\end{array}$ \\
\hline & & $540 \mathrm{~cm}^{-1}(67 \mathrm{meV})$ & $\begin{array}{l}\text { dipole allowed mode [this } \\
\text { work] }\end{array}$ \\
\hline
\end{tabular}

however, often mixtures of the crystalline and vitreous forms of the bilayer. In light of a recent report, which suggests that vibrational spectroscopy may be used to discriminate between the two forms of the film, these two forms are compared first. ${ }^{19}$ The black trace in Figure 1 shows the HREELS spectrum of a silica bilayer film. The LEED pattern (Figure 1a) exhibits sharp hexagonal diffraction spots and no indication for a diffuse ring structure, which is indicative of a largely crystalline nature of this preparation. The corresponding HREELS spectrum is dominated by two phonon signals of essentially the same intensity at $681 \mathrm{~cm}^{-1}(84.4 \mathrm{meV})$ and $1278 \mathrm{~cm}^{-1}(158.5$ $\mathrm{meV}$ ). The line positions are in line with the ones observed by IR spectroscopy. ${ }^{13}$ Upon annealing of this film for $10 \mathrm{~min}$ under UHV conditions to $1023 \mathrm{~K}$, the sharp hexagonal diffraction spots found for a crystalline film were converted into a diffusive ring structure (Figure $1 \mathrm{~b}$ ). This ring structure is indicative for the presence of a vitreous film, and no indications for remaining crystalline areas can be found in the LEED image. The HREELS measurements show that the transition from the crystalline (Figure 1, black curve) to vitreous form (Figure 1, blue curve) by annealing under UHV conditions has only very little influence on the observed phonon spectrum.

The two main phonon signals were found at almost the same position with the same line shape and width. The intensity of the low-energy phonon at $681 \mathrm{~cm}^{-1}$ was found to be slightly enhanced relative to the one at $1278 \mathrm{~cm}^{-1}$. The similarity between the spectra of the crystalline and vitreous forms of the film (black and blue trace in Figure 1) is not only found for the main lines but also extends to the smaller signals (see inset of Figure 1). Except for small changes in the relative intensities found systematically for all low-energy phonons $\left(<681 \mathrm{~cm}^{-1}\right)$, the largest change concerns the low-energy shoulder around $1200 \mathrm{~cm}^{-1}$ and the signals at $1054(130.7 \mathrm{meV})$ and $977 \mathrm{~cm}^{-1}$ $(121.1 \mathrm{meV})$, which lose intensity upon annealing the film to prepare the vitreous form; however, some of the decrease observed for the signals at 1054 and $977 \mathrm{~cm}^{-1}$ may be due to the reduction in the intensity of the broad shoulder on the lowenergy side of the main peak at $1278 \mathrm{~cm}^{-1}$. Beside these signals, the spectra exhibit a set of additional lines with low intensity. The most prominent one is at $562 \mathrm{~cm}^{-1}(69.7 \mathrm{meV})$, and additional signals are found at $429 \mathrm{~cm}^{-1}(53.2 \mathrm{meV}), 483$ $\mathrm{cm}^{-1}$ (59.9 meV), $838 \mathrm{~cm}^{-1}(103.9 \mathrm{meV}), 905 \mathrm{~cm}^{-1}(112.2$ $\mathrm{meV})$, and $1366 \mathrm{~cm}^{-1}(169.4 \mathrm{meV})$. The small signal at 1366 $\mathrm{cm}^{-1}(169.4 \mathrm{meV})$ located on the high-energy side of the phonon at $1278 \mathrm{~cm}^{-1}$ is due to double excitation of the phonon signal at $681 \mathrm{~cm}^{-1}$. A summary of all signals is found in Table 1. A comparison with isotopically labelled $\mathrm{Si}^{18} \mathrm{O}_{2}$ films shows the expected shift to lower vibrational frequency because of the change in reduced mass, indicating that these peaks are related to oxygen-related vibrations (Supporting Information).

The weak lines, which were not observed in the IR spectra, may be in part observed in the HREELS spectra because of the excitation of dipole-forbidden modes by impact scattering. To explore this effect, a HREELS spectrum taken in the offspecular geometry (dark blue) is compared to the one obtained in the specular geometry (light blue) in Figure 2. Because of the strongly focused intensity distribution along the specular direction expected for dipole scattering, a significant relative increase of loss intensity at a nonspecular angle points to an excitation involving impact scattering. Such an increase is observed for most of the small signals present in the spectrum except for the bands at 562 and $905 \mathrm{~cm}^{-1}$. A broad maximum at about $438 \mathrm{~cm}^{-1}(54.3 \mathrm{meV})$ is only visible in the off-specular spectrum, which is a strong indication that this mode is dipoleforbidden. These results clearly indicate that the HREELS spectra allow to observe non-dipole-active modes of the silica double layer, which cannot be observed by IR spectroscopy.

As evident from the complete list of normal modes expected for a silica bilayer (see the Supporting Information), the presence of additional lines is expected, and the observed signals are well in line with the predicted vibrations of the system. However, a direct assignment of the signals to individual normal modes is hampered by the complexity to predict their cross-section with respect to impact scattering. Two-dimensional silica bilayer structures have been investigated using Raman spectroscopy and DFT calculations. ${ }^{19}$ 


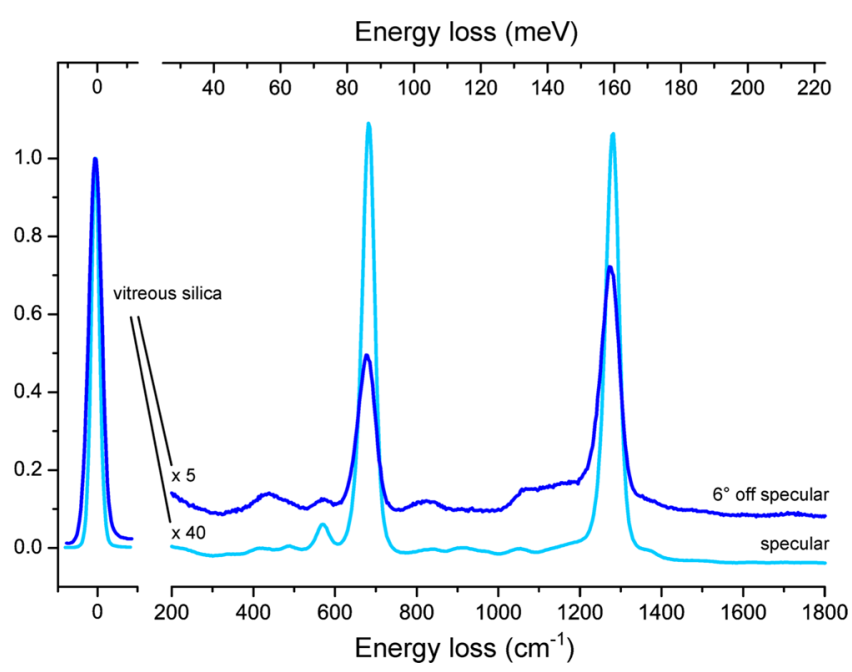

Figure 2. Angular-dependent HREELS spectra of a vitreous silica film on $\mathrm{Ru}(0001)$.

The most prominent peak found in the Raman spectra was observed at $1045 \mathrm{~cm}^{-1}(129.6 \mathrm{meV})$ and was assigned to the Raman-active asymmetric stretching mode of a Kagome lattice (the hexagonal crystalline form of the film) with a calculated frequency of $1053 \mathrm{~cm}^{-1}(130.6 \mathrm{meV})$. The signal fits nicely to the corresponding signal in the HREELS spectra at $1054 \mathrm{~cm}^{-1}$. The DFT calculations show several modes around this energy, which show basically no transition dipole moment, in line with the expected mutual exclusion of the Raman- and IR-active modes for systems with inversion symmetry. The presence of the signal in the HREELS spectra and its relative enhancement in the spectra taken in the off-specular geometry fit into this picture. The Raman spectrum exhibits additional small signals at $409 \mathrm{~cm}^{-1}(50.7 \mathrm{meV}), 454 \mathrm{~cm}^{-1}(56.3 \mathrm{meV})$, and $500 \mathrm{~cm}^{-1}$ $(62.0 \mathrm{meV})$. The HREELS spectra show signals at similar frequencies (429 and $483 \mathrm{~cm}^{-1}$ ), however, a direct correlation with the Raman spectra is hampered because of a large number of possible modes in this frequency range predicted by DFT calculations (see the Supporting Information). The difficulty to properly assign these lines is in line with previous theoretical calculations reported by Björkman et al. ${ }^{19}$

As shown in Figure 1, the crystalline and vitreous silica bilayers exhibit very similar spectra, which renders discrimination of the two allotropes impossible within the resolution of the HREELS spectra. Please note that HREELS is also capable of detecting non-dipolar-active modes as discussed above, which shows that the similarity is not restricted to the dipole-active modes. The similarity of the HREELS spectra of the two allotropes indicates that the position of the phonon modes is rather insensitive to the binding angles in the $\mathrm{SiO}_{4}$ tetrahedra, which vary between the six-membered rings found for the crystalline and the four- to nine-membered rings observed for the vitreous film. ${ }^{18}$ However, one should keep in mind that the most common ring type in the vitreous film is still the six-membered ring that is found to have a very similar in-plane $\mathrm{Si}-\mathrm{Si}-\mathrm{Si}$ angle of $118.8^{\circ}$ versus $119.5^{\circ}$ for the crystalline phase. ${ }^{18} \mathrm{With}$ respect to the dipole-active modes as well as the main Raman signal at $1053 \mathrm{~cm}^{-1}$, the observation is in line with theoretical efforts by Björkman et al., ${ }^{19}$ who compared the vibrational frequencies of the hexagonal crystalline form to those of a simple model (the so-called haeckelite structure) for the vitreous phase. These calculations show that the IR-active signals as well as the main Raman signal at $1045 \mathrm{~cm}^{-1}$ are insensitive to the lateral organization of the $\mathrm{SiO}_{2}$ films, whereas there was more spread in the calculated vibrational frequencies for low-energy vibrations. The reduction in symmetry also lifts the selection rules (loss of mutual exclusion of Raman and IR signals in the absence of a center of inversion) and gives rise to more complex eigenvectors of the corresponding normal modes, which might enable detection by vibrational spectroscopy. ${ }^{19}$ However, this effect of symmetry reduction does not lead to any clearly visible effects in the HREELS spectra of the silica bilayer.

With respect to a proper assignment of the various signals observed in the HREELS spectra, it is important to note that the intensity of those not yet discussed depends on the preparation condition. A first example was already described above (Figure 1), which had revealed a loss of intensity on the low-energy side of the main signal at $1278 \mathrm{~cm}^{-1}$. Signals in this range were previously observed by IRAS for films beyond the bilayer coverage. For a 4 MLE thick film, a new signal was observed at $1257 \mathrm{~cm}^{-1}$, which exhibits a broad shoulder down to $1164 \mathrm{~cm}^{-1}$. $^{12}$ Similar signals were observed between 1150 and $1250 \mathrm{~cm}^{-1}$ for nanometer thick, amorphous silica films on different Mo surfaces as well as on $\mathrm{Si}(100))^{3,4,44-50}$ On the basis of these results, we assign the low-energy shoulder of the phonon at $1278 \mathrm{~cm}^{-1}$ (Figure 1) to the presence of threedimensional (3D) particles grown on the silica bilayer because of a slightly higher coverage than 2 MLE. The reduction of the corresponding intensity after annealing the system indicates the removal of these features from the surface, in line with previous results. ${ }^{12}$ LEED experiments have shown that the film starts to dewet if annealed above about $1200 \mathrm{~K}$. As a result, holes of oxygen-covered $\mathrm{Ru}(0001)$ as well as areas covered with a monolayer silica film were observed. ${ }^{51}$ The latter is characterized by a signal observed at $1134 \mathrm{~cm}^{-1}$ by IR spectroscopy, which is compatible with the small peak at around $1120 \mathrm{~cm}^{-1}$ (Figure 1, blue trace). ${ }^{7,12,17,18}$

Figure 3 shows the HREELS spectrum of a monolayer $\mathrm{SiO}_{2}$ film (orange trace) prepared by depositing $50 \%$ of the amount of silicon (1 MLE Si) used for the bilayer and keeping the other parameters unchanged, which was previously shown to result in a monolayer. ${ }^{12,52}$ The monolayer consists of a single layer of six-membered rings, which are connected to the support by covalent bonds between oxygen and $\mathrm{Ru}(0001)$ underneath.

In comparison to the monolayer IRAS spectra characterized by peaks at $1134,1074,790$, and $687 \mathrm{~cm}^{-1}$, the lines observed in the HREELS spectrum are slightly shifted by $6-14 \mathrm{~cm}^{-1}{ }^{12}$ Apart from these, the HREELS spectrum of the monolayer exhibits small signals at $886,970,463$, and $308 \mathrm{~cm}^{-1}$ as well as a shoulder at about $1200 \mathrm{~cm}^{-1}$. The presented HREELS spectrum of the monolayer film is in very good agreement with the calculated vibrational modes indicated by vertical lines in Figure 3. The agreement between experiment and quantum mechanical calculations extends beyond the range accessible by IR, namely, below $680 \mathrm{~cm}^{-1}$. The HREELS spectrum allows to identify a vibration at $308 \mathrm{~cm}^{-1}$ as well as $463 \mathrm{~cm}^{-1}$, which fit nicely to the calculated spectrum. However, the signal around $540 \mathrm{~cm}^{-1}$ predicted by DFT is not prominently found in the HREELS spectrum. As indicated by the vertical lines, the signals at 1121 and $1060 \mathrm{~cm}^{-1}$ are also observed in the spectrum of a bilayer (purple trace). A comparison of the relative intensities of the lines at 1121 and $1060 \mathrm{~cm}^{-1}$ of the monolayer and bilayer reveals that the signal at $1060 \mathrm{~cm}^{-1}$ 


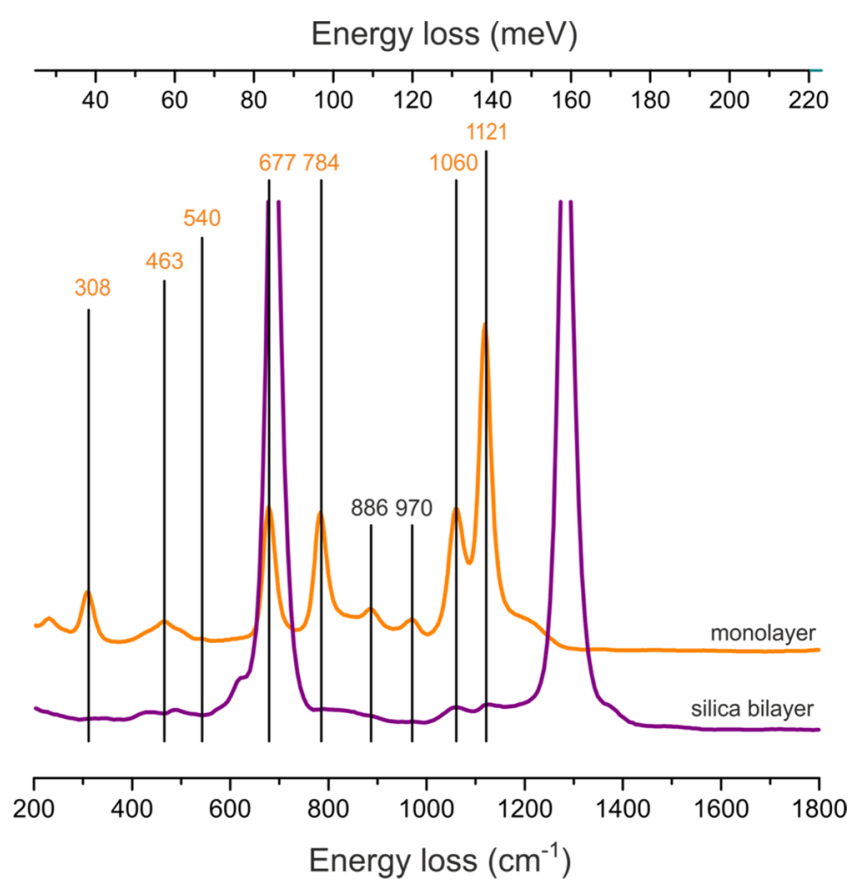

Figure 3. HREELS spectra of a silica bilayer film on $\mathrm{Ru}(0001)$ (purple trace) and a monolayer film on $\mathrm{Ru}(0001)$ (orange trace). The vertical lines marked with orange numbers refer to vibrational modes also predicted by DFT calculations (see also Table S2). ${ }^{15}$

observed in the bilayer cannot solely be attributed to some fraction of the surface covered with the silica monolayer, which corroborates the assignment of the line around $1060 \mathrm{~cm}^{-1}$ to be at least partially due to a non-dipole-active mode of the bilayer. In addition, the spectrum of the monolayer exhibits two bands at 886 and $970 \mathrm{~cm}^{-1}$, which are not predicted by DFT calculations to be part of the monolayer spectrum but have been observed in bulk silica materials. ${ }^{44,50}$ In combination with the presence of the shoulder around $1200 \mathrm{~cm}^{-1}$, which was also observed for some bilayer preparations, these signals can be assigned to small silica particles residing on top of the monolayer film. ${ }^{51}$

A prominent feature of the HREELS spectra of crystalline and vitreous silica bilayer films is a peak at $562 \mathrm{~cm}^{-1}(69.7$
$\mathrm{meV}$ ), which was shown to be a dipole-active mode (Figure 2). In the spectral range between $539 \mathrm{~cm}^{-1}(66.8 \mathrm{meV})$ and 646 $\mathrm{cm}^{-1}(80.1 \mathrm{meV})$, vibrational modes of oxygen adsorbed on $\mathrm{Ru}(0001)$ were previously observed. ${ }^{24,25}$ The presence of oxygen atoms on $\mathrm{Ru}(0001)$ underneath the silica bilayer is possible because of the weak interaction between the coordinatively saturated film and the metal surface. Intercalation experiments have shown that the oxygen coverage of ruthenium can be varied and plays a decisive role for the reaction behavior of this system; hence, it is interesting to investigate if the adsorbed oxygen atoms can be characterized using vibrational spectroscopy. ${ }^{21}$ The adsorption structure of $\mathrm{O}$ atoms on the $\mathrm{Ru}(0001)$ surface underneath the silica bilayer depends rather crucially on details of the preparation procedure. To illustrate this effect, Figure 4a compares the HREELS spectrum of two silica films, which were prepared using nominally the same preparation procedure. In the region below the main phonon around $650 \mathrm{~cm}^{-1}$, both preparations exhibit signals at $429 \mathrm{~cm}^{-1}$ and $483 \mathrm{~cm}^{-1}$. However, the signal at $564 \mathrm{~cm}^{-1}$ observed for the green trace is missing in the purple spectrum. Instead the latter spectrum exhibits a shoulder at around $620 \mathrm{~cm}^{-1}$, which is not found in the green spectrum.

The oxygen coverages at the interface of a silica bilayer film supported on $\mathrm{Ru}(0001)$ can be reversibly altered by annealing the film to $1000 \mathrm{~K}$ in an oxygen atmosphere $\left(p\left(\mathrm{O}_{2}\right)=3 \times 10^{-6}\right.$ mbar) and under UHV conditions. Figure $4 \mathrm{~b}$ presents the spectra of a silica bilayer film annealed to $1000 \mathrm{~K}$ under UHV conditions (dark gray trace) compared to the film upon annealing in the oxygen atmosphere (purple trace) and subsequent annealing in UHV (light gray trace). The UHVannealed sample exhibits, apart from two signals at $429 \mathrm{~cm}^{-1}$ $(53.2 \mathrm{meV})$ and $483 \mathrm{~cm}^{-1}(59.9 \mathrm{meV})$ and the main phonon line present in all spectra, a signal at $574 \mathrm{~cm}^{-1}$ slightly blueshifted compared to the spectra discussed above. After annealing in oxygen, this signal disappears, and a shoulder around $624 \mathrm{~cm}^{-1}$ appears indicating that this feature corresponds to a higher oxygen coverage. Subsequent annealing in UHV depletes the shoulder and restores the signal at $574 \mathrm{~cm}^{-1}$. However, the signal at $574 \mathrm{~cm}^{-1}$ is broadened at the high-energy side as compared to the dark gray trace at the bottom. This procedure illustrates the
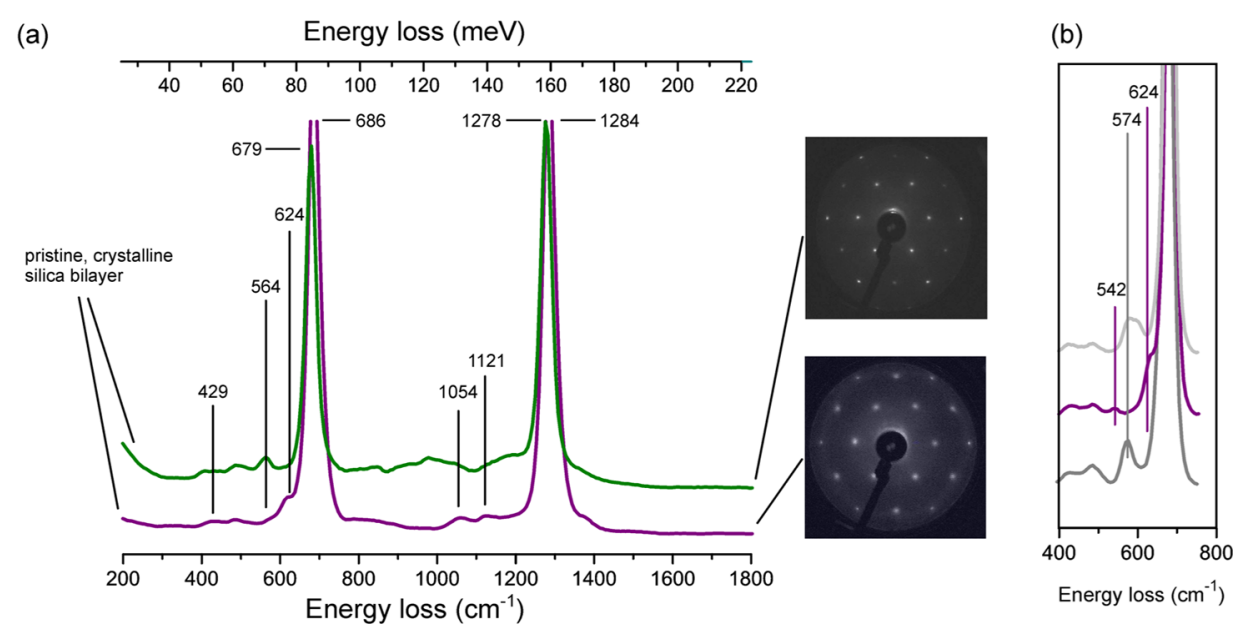

Figure 4. HREELS spectra of two pristine, crystalline silica bilayer films (purple and green trace) (a). HREELS spectra of the $\mathrm{SiO}_{2}$ bilayer on $\mathrm{Ru}$ (0001) with different oxygen coverages at the interface (b): annealing under UHV conditions (dark gray trace), annealing in oxygen (purple trace), and annealing under UHV conditions (light gray trace). 
reversible change of the oxygen coverage on the $\mathrm{Ru}(0001)$ surface, and according to the preparation procedure, the signal observed at $574 \mathrm{~cm}^{-1}$ should correspond to an oxygen poor (O-poor) situation, whereas the shoulder around $620 \mathrm{~cm}^{-1}$ can be assigned to an oxygen rich (O-rich) situation. This process was previously investigated by X-ray photoelectron spectroscopy (XPS) showing a reversible formation of the so-called Opoor and O-rich phases between $\mathrm{SiO}_{2}$ and $\mathrm{Ru}(0001)$ by annealing to $1140 \mathrm{~K}$ in $2 \times 10^{-6} \mathrm{mbar}$ of $\mathrm{O}_{2}{ }^{20}$ In combination with DFT calculations, it was shown that the amount of interfacial oxygen depends on the annealing temperature. An oxygen coverage of $0.75 \mathrm{ML}[3 \mathrm{O}(2 \times 2) \mathrm{Ru}]$ was calculated to be the most stable structure around $900 \mathrm{~K}$; at $1000 \mathrm{~K}$, the $\mathrm{O}(2$ $\times 1)$ and $3 \mathrm{O}(2 \times 2)$ structures have a similar stability. Temperature-programmed desorption experiments reveal that the desorption temperature of oxygen adsorbed on $\mathrm{Ru}(0001)$ lowers with increasing oxygen coverage, with a desorption temperature of $1100 \mathrm{~K}$ for $\mathrm{O}(2 \times 1)$ on $\mathrm{Ru}(0001))^{30,53,54}$ Because of the complex phase behavior, the experimental results are compared to predictions from DFT calculations. The calculated vibrational spectrum of the $3 \mathrm{O}(2 \times 2)$ structure on $\mathrm{Ru}(0001)$ underneath the silica bilayer results in a complex multiline spectrum, with the most intense peak red-shifted by $67 \mathrm{~cm}^{-1}$ relative to the main phonon line (Figure S3). The calculated spectrum of the $(2 \times 1)$ structure (Figure S3) shows a single line blue-shifted by $27 \mathrm{~cm}^{-1}$ with respect to the main signal of the $3 \mathrm{O}(2 \times 2)$ oxygen superstructure, which is in contradiction with the experimental results showing a blue shift of the line with increasing oxygen coverage (Figure $4 b$ ). Thus, these two situations cannot serve as an explanation for the experimental results. The signal calculated for an oxygen coverage of $\theta=0.25$ in the $\mathrm{SiO}_{2} / \mathrm{Ru}(0001)$ system (Figure S3) is red-shifted with respect to both high oxygen-coverage situations and allows to interpret the experimentally observed red shift upon reduction of oxygen coverage on the Ru surface. However, it is significantly more red-shifted than that observed experimentally, which might be due to a somewhat higher oxygen coverage present under the experimental conditions. ${ }^{25}$ On the basis of this comparison, the oxygen-rich phase would be assigned to the $(2 \times 1)$ oxygen phase with an oxygen coverage $\theta$ of about 0.5 , whereas annealing in UHV results in a reduction of the oxygen coverage, which remains above $\theta=$ 0.25 and is consistent with the assignment made before based on XPS results. ${ }^{23}$ Please note that the vibrational spectrum of oxygen atoms on $\mathrm{Ru}(0001)$ is significantly altered by the presence of the silica bilayer despite the weak interaction between the film and the metal surface (Figures S2 and S3), which would cause erroneous assignments of the lines, if the experimental signals obtained from the $\mathrm{Ru}(0001)$ surface were used for assignment. The statement is further supported by a slight blue shift of the main phonons $\left(6-7 \mathrm{~cm}^{-1}\right)$ observed for the oxygen-rich superstructure (purple spectrum, Figure $4 a$ ). This shift qualitatively concurs with the DFT results (Figure S3). However, the experimentally observed shift is somewhat larger. ${ }^{20}$

\section{CONCLUSIONS}

In summary, we have shown that a combination of HREELS and DFT calculations allows for an in-depth analysis of the vibrational properties of silica bilayer films grown on $\mathrm{Ru}(0001)$. In particular, it has been shown that it is possible to investigate dipole-inactive vibrations using HREELS and to assign some of the observed signals not present in IR spectra to the dipole-inactive modes of the silica bilayer. The modes at 1060,424 , and $483 \mathrm{~cm}^{-1}$ could be correlated with the bilayer structure, in line with previous results based on Raman spectroscopy. The HREELS spectra of the two allotropes of the film, namely, the crystalline and vitreous forms, show virtually no difference in the vibrational properties with respect to the signal position, line shape, and width. Thus, the experimental results of this study give no support for the claim of Björkman et al. ${ }^{19}$ that differences between the low-energy phonons of the two silica phases might be used to differentiate between them.

By a combination of theory and results from the literature, it was shown that some of the signals, whose intensity varies considerably with details of the preparation, are due to additional silica particles or small amounts of monolayer films, depending on the precise amount of deposited $\mathrm{Si}$ as well as on the details of the annealing procedure.

Finally, HREELS allows to address the vibrational properties of oxygen atoms adsorbed on the $\mathrm{Ru}(0001)$ surface beneath the silica film. The oxygen coverage can be altered reversibly by annealing the film to $1000 \mathrm{~K}$ in UHV or an oxygen atmosphere, which leads to characteristic changes of the corresponding signals. An interpretation of these changes requires theoretical support, as the presence of the silica film leads to a significant alteration of signal positions as compared to the bare oxygen-covered metal surface. By comparison with the theoretical calculation, the "oxygen-rich" situation could be assigned to an oxygen coverage of about $0.5 \mathrm{ML}$, whereas reduction leads to an oxygen coverage above $0.25 \mathrm{ML}$. With respect to the range of signals present in the HREELS spectra below the main high-energy phonon line, it was possible to assign some of them to small amounts of monolayer patches present in the preparation in different quantities. To this end, the HREELS spectra nicely confirm the theoretical predictions for the vibrational spectrum of the monolayer also below 680 $\mathrm{cm}^{-1}$.

\section{ASSOCIATED CONTENT}

\section{Supporting Information}

The Supporting Information is available free of charge on the ACS Publications website at DOI: 10.1021/acs.jpcc.8b10478.

Comparison of HREELS spectra taken for preparation with ${ }^{16} \mathrm{O}_{2}$ and ${ }^{18} \mathrm{O}_{2}$, comparison of the vibrational modes obtained from the experiment and theory, computational details for the calculation of vibrational frequencies as well as calculated vibrational spectra for different oxygen coverages on the $\mathrm{Ru}(0001)$ surface and on $\mathrm{Ru}(0001)$ beneath the silica bilayer, and atomic coordinates as well as a full list of the vibrational modes of the $\mathrm{SiO}_{2}-3 \mathrm{O}(2$ $\times 2) \mathrm{Ru}(0001)$ structure (PDF)

\section{AUTHOR INFORMATION}

\section{Corresponding Authors}

*E-mail: kuhlenbeck@fhi-berlin.mpg.de (H.K.).

*E-mail: risse@chemie.fu-berlin.de (T.R.).

\section{ORCID}

Shamil Shaikhutdinov: 0000-0001-9612-9949

Thomas Risse: 0000-0003-0228-9189

Hans-Joachim Freund: 0000-0001-5188-852X

Joachim Sauer: 0000-0001-6798-6212 


\section{Notes}

The authors declare no competing financial interest.

\section{ACKNOWLEDGMENTS}

We acknowledge the support from DFG through the collaborative research program SFB 1109 . We cordially thank Matthias Naschitzki for the technical support and assistance. N.F.R. thanks the International Max-Planck Research School "Functional interfaces in physics and chemistry."

\section{REFERENCES}

(1) He, J.-W.; Xu, X.; Corneille, J. S.; Goodman, D. W. X-ray Photoelectron Spectroscopic Characterization of Ultra-thin Silicon Oxide Films on a Mo(100) Surface. Surf. Sci. 1992, 279, 119-126.

(2) Giordano, L.; Ricci, D.; Pacchioni, G.; Ugliengo, P. Structure and Vibrational Spectra of Crystalline $\mathrm{SiO}_{2}$ Ultra-thin Films on Mo(112). Surf. Sci. 2005, 584, 225-236.

(3) Xu, X.; Goodman, D. W. New Approach to the Preparation of Ultrathin Silicon Dioxide Films at low Temperatures. Appl. Phys. Lett. 1992, 61, 774-776.

(4) Xu, X.; Goodman, D. W. The Preparation and Characterization of Ultra-thin Silicon Dioxide Films on a Mo(110) Surface. Surf. Sci. 1993, 282, 323-332.

(5) Zhang, Z.; Jiang, Z.; Yao, Y.; Tan, D.; Fu, Q.; Bao, X. Preparation and Characterization of Atomically Flat and Ordered Silica Films on a Pd(100) Surface. Thin Solid Films 2008, 516, 3741-3746.

(6) Schroeder, T.; Adelt, M.; Richter, B.; Naschitzki, M.; Bäumer, M.; Freund, H.-J. Growth of Well-Ordered Silicon Dioxide Films on Mo(112). Microelectron. Reliab. 2000, 40, 841-844.

(7) Lichtenstein, L.; Büchner, C.; Yang, B.; Shaikhutdinov, S.; Heyde, M.; Sierka, M.; Włodarczyk, R.; Sauer, J.; Freund, H.-J. The Atomic Structure of a Metal-Supported Vitreous Thin Silica Film. Angew. Chem., Int. Ed. 2012, 51, 404-407.

(8) Shaikhutdinov, S.; Freund, H.-J. Ultrathin Silica Films on Metals: The Long and Winding Road to Understanding the Atomic Structure. Adv. Mater. 2013, 25, 49-67.

(9) Büchner, C.; Heyde, M. Two-dimensional silica opens new perspectives. Prog. Surf. Sci. 2017, 92, 341-374.

(10) Weissenrieder, J.; Kaya, S.; Lu, J.-L.; Gao, H.-J.; Shaikhutdinov, S.; Freund, H.-J.; Sierka, M.; Todorova, T. K.; Sauer, J. Atomic Structure of a Thin Silica Film on a Mo(112) Substrate: A TwoDimensional Network of $\mathrm{SiO}_{4}$ Tetrahedra. Phys. Rev. Lett. 2005, 95, 076103.

(11) Lu, J.-L.; Kaya, S.; Weissenrieder, J.; Todorova, T. K.; Sierka, M.; Sauer, J.; Gao, H.-J.; Shaikhutdinov, S.; Freund, H.-J. Formation of One-Dimensional Crystalline Silica on a Metal Substrate. Surf. Sci. Lett. 2006, 600, L164-L168.

(12) Yang, B.; Kaden, W. E.; Yu, X.; Boscoboinik, J. A.; Martynova, Y.; Lichtenstein, L.; Heyde, M.; Sterrer, M.; Włodarczyk, R.; Sierka, M.; et al. Thin Silica Films on $\mathrm{Ru}(0001)$ : Monolayer, Bilayer and Threedimensional Network of $\left[\mathrm{SiO}_{4}\right]$ Tetrahedra. Phys. Chem. Chem. Phys. 2012, 14, 11344-11351.

(13) Löffler, D.; Uhlrich, J. J.; Baron, M.; Yang, B.; Yu, X.; Lichtenstein, L.; Heinke, L.; Büchner, C.; Heyde, M.; Shaikhutdinov, S.; et al. Growth and Structure of Crystalline Silica Sheet on $\mathrm{Ru}(0001)$. Phys. Rev. Lett. 2010, 105, 146104.

(14) Yu, X.; Yang, B.; Anibal Boscoboinik, J.; Shaikhutdinov, S.; Freund, H.-J. Support Effects on the Atomic Structure of Ultrathin Silica Films on Metals. Appl. Phys. Lett. 2012, 100, 151608.

(15) Crampton, A. S.; Ridge, C. J.; Rötzer, M. D.; Zwaschka, G.; Braun, T.; D’Elia, V.; Basset, J.-M.; Schweinberger, F. F.; Günther, S.; Heiz, U. Atomic Structure Control of Silica Thin Films on Pt(111). J. Phys. Chem. C 2015, 119, 13665-13669.

(16) Jhang, J.-H.; Zhou, C.; Dagdeviren, O. E.; Hutchings, G. S.; Schwarz, U. D.; Altman, E. I. Growth of Two Dimensional Silica and Aluminosilicate Bilayers on $\operatorname{Pd}(111)$ : From Incommensurate to Commensurate Crystalline. Phys. Chem. Chem. Phys. 2017, 19, 14001-14011.
(17) Lichtenstein, L.; Heyde, M.; Freund, H.-J. Crystalline-Vitreous Interface in Two Dimensional Silica. Phys. Rev. Lett. 2012, 109, 106101.

(18) Lichtenstein, L.; Heyde, M.; Freund, H.-J. Atomic Arrangement in Two-Dimensional Silica: From Crystalline to Vitreous Structures. J. Phys. Chem. C 2012, 116, 20426-20432.

(19) Björkman, T.; Skakalova, V.; Kurasch, S.; Kaiser, U.; Meyer, J. C.; Smet, J. H.; Krasheninnikov, A. V. Vibrational Properties of a Two-Dimensional Silica Kagome Lattice. ACS Nano 2016, 10, 10929-10935.

(20) Włodarczyk, R.; Sierka, M.; Sauer, J.; Löffler, D.; Uhlrich, J. J.; Yu, X.; Yang, B.; Groot, I. N. M.; Shaikhutdinov, S.; Freund, H.-J. Tuning the Electronic Structure of Ultrathin Crystalline Silica Films on $\mathrm{Ru}(0001)$. Phys. Rev. B: Condens. Matter Mater. Phys. 2012, 85, 085403.

(21) Emmez, E.; Yang, B.; Shaikhutdinov, S.; Freund, H.-J. Permeation of a Single-Layer $\mathrm{SiO}_{2}$ Membrane and Chemistry in Confined Space. J. Phys. Chem. C 2014, 118, 29034-29042.

(22) Kaden, W. E.; Büchner, C.; Lichtenstein, L.; Stuckenholz, S.; Ringleb, F.; Heyde, M.; Sterrer, M.; Freund, H.-J. Understanding Surface Core-Level Shifts Using the Auger Parameter: A Study of Pd Atoms Adsorbed on Ultrathin $\mathrm{SiO}_{2}$ Films. Phys. Rev. B: Condens. Matter Mater. Phys. 2014, 89, 115436.

(23) Prieto, M. J.; Klemm, H. W.; Xiong, F.; Gottlob, D. M.; Menzel, D.; Schmidt, T.; Freund, H.-J. Water Formation under Silica Thin Films: Real-Time Observation of a Chemical Reaction in a Physically Confined Space. Angew. Chem., Int. Ed. 2018, 57, 8749-8753.

(24) Mitchell, W. J.; Wang, Y.; Schick, M.; Weinberg, W. H. Surface Phonons on $\mathrm{Ru}(001)$ Observed by Electron Energy Loss Spectroscopy in the Presence of Ordered Oxygen Overlayers. J. Chem. Phys. 1995, 102, 8185-8190.

(25) Kostov, K. L.; Gsell, M.; Jakob, P.; Moritz, T.; Widdra, W.; Menzel, D. Observation of a novel high density $3 \mathrm{O}(2 \times 2)$ structure on $\mathrm{Ru}(001)$. Surf. Sci. Lett. 1997, 394, L138-L144.

(26) He, P.; Jacobi, K. Vibrational Analysis of the $1 \times 1-\mathrm{O}$ Overlayer on $\mathrm{Ru}(0001)$. Phys. Rev. B: Condens. Matter Mater. Phys. 1997, 55, 4751-4754.

(27) Gsell, M.; Stichler, M.; Jakob, P.; Menzel, D. Formation and Geometry of a High-Coverage Oxygen Adlayer on $\mathrm{Ru}(001)$, the $\mathrm{p}(2$ $\times 2)$-3O Phase. Isr. J. Chem. 1998, 38, 339-348.

(28) Kim, Y. D.; Wendt, S.; Schwegmann, S.; Over, H.; Ertl, G. Structural Analyses of the Pure and Cesiated $\mathrm{Ru}(0001)-(2 \times 2)-3 \mathrm{O}$ Phase. Surf. Sci. 1998, 418, 267-272.

(29) Moritz, T.; Menzel, D.; Widdra, W. Collective Vibrational Modes of the High-Density (1×1)-O Phase on $\mathrm{Ru}(001)$. Surf. Sci. 1999, 427-428, 64-68.

(30) Madey, T. E.; Albert Engelhardt, H.; Menzel, D. Adsorption of Oxygen and Oxidation of CO on the Ruthenium (001) Surface. Surf. Sci. 1975, 48, 304-328.

(31) Büchner, C.; Eder, S. D.; Nesse, T.; Kuhness, D.; Schlexer, P.; Pacchioni, G.; Manson, J. R.; Heyde, M.; Holst, B.; Freund, H. J. Bending Rigidity of 2D Silica. Phys. Rev. Lett. 2018, 120, 226101.

(32) Kresse, G.; Furthmüller, J. Efficient Iterative Schemes for ab initio Total-Energy Calculations Using a Plane-Wave Basis Set. Phys. Rev. B: Condens. Matter Mater. Phys. 1996, 54, 11169-11186.

(33) Kresse, G.; Furthmüller, J. Efficiency of ab-initio Total Energy Calculations for Metals and Semiconductors Using a Plane-Wave Basis Set. Comput. Mater. Sci. 1996, 6, 15-50.

(34) Perdew, J. P.; Burke, K.; Ernzerhof, M. Generalized Gradient Approximation Made Simple. Phys. Rev. Lett. 1996, 77, 3865-3868.

(35) Perdew, J. P.; Burke, K.; Ernzerhof, M. Generalized Gradient Approximation Made Simple [Phys. Rev. Lett. 77, 3865 (1996)]. Phys. Rev. Lett. 1997, 78, 1396.

(36) Blöchl, P. E. Projector Augmented-Wave Method. Phys. Rev. B: Condens. Matter Mater. Phys. 1994, 50, 17953-17979.

(37) Kresse, G.; Joubert, D. From Ultrasoft Pseudopotentials to the Projector Augmented-Wave Method. Phys. Rev. B: Condens. Matter Mater. Phys. 1999, 59, 1758-1775. 
(38) Grimme, S. Accurate Description of van der Waals Complexes by Density Functional Theory Including Empirical Corrections. J. Comput. Chem. 2004, 25, 1463-1473.

(39) Grimme, S. Semiempirical Hybrid Density Functional with Perturbative Second-Order Correlation. J. Chem. Phys. 2006, 124, 034108 .

(40) Kerber, T.; Sierka, M.; Sauer, J. Application of Semiempirical Long-Range Dispersion Corrections to Periodic Systems in Density Functional Theory. J. Comput. Chem. 2008, 29, 2088-2097.

(41) Monkhorst, H. J.; Pack, J. D. Special Points for Brillouin-Zone Integrations. Phys. Rev. B: Condens. Matter Mater. Phys. 1976, 13, $5188-5192$.

(42) Scott, J. F.; Porto, S. P. S. Longitudinal and Transverse Optical Lattice Vibrations in Quartz. Phys. Rev. 1967, 161, 903-910.

(43) Sato, R. K.; McMillan, P. F. An Infrared and Raman Study of the Isotopic Species of $\alpha$-Quartz. J. Phys. Chem. 1987, 91, 3494-3498.

(44) Thiry, P. A.; Liehr, M.; Pireaux, J. J.; Sporken, R.; Caudano, R.; Vigneron, J. P.; Lucas, A. A. Vibrational Study of the $\mathrm{SiO}_{2} / \mathrm{Si}$ Interface by High Resolution Electron Energy Loss Spectroscopy. J. Vac. Sci. Technol., B 1985, 3, 1118-1121.

(45) Kirk, C. T. Quantitative Analysis of the Effect of DisorderInduced Mode Coupling on Infrared Absorption in Silica. Phys. Rev. B: Condens. Matter Mater. Phys. 1988, 38, 1255-1273.

(46) Queeney, K. T.; Weldon, M. K.; Chang, J. P.; Chabal, Y. J.; Gurevich, A. B.; Sapjeta, J.; Opila, R. L. Infrared Spectroscopic Analysis of the $\mathrm{Si} / \mathrm{SiO}_{2}$ Interface Structure of Thermally Oxidized Silicon. J. Appl. Phys. 2000, 87, 1322-1330.

(47) Schroeder, T.; Giorgi, J. B.; Bäumer, M.; Freund, H.-J. Morphological and Electronic Properties of Ultrathin Crystalline Silica Epilayers on a Mo(112) Substrate. Phys. Rev. B: Condens. Matter Mater. Phys. 2002, 66, 165422.

(48) Queeney, K. T.; Herbots, N.; Shaw, J. M.; Atluri, V.; Chabal, Y. J. Infrared Spectroscopic Analysis of an Ordered $\mathrm{Si} / \mathrm{SiO}_{2}$ Interface. Appl. Phys. Lett. 2004, 84, 493-495.

(49) Wendt, S.; Ozensoy, E.; Wei, T.; Frerichs, M.; Cai, Y.; Chen, M. S.; Goodman, D. W. Electronic and Vibrational Properties of Ultrathin $\mathrm{SiO}_{2}$ Films Grown on $\mathrm{Mo}(112)$. Phys. Rev. B: Condens. Matter Mater. Phys. 2005, 72, 115409.

(50) Todorova, T. K.; Sierka, M.; Sauer, J.; Kaya, S.; Weissenrieder, J.; Lu, J. L.; Gao, H. J.; Shaikhutdinov, S.; Freund, H. J. Atomic Structure of a Thin Silica Film on a Mo(112) Substrate: A Combined Experimental and Theoretical Study. Phys. Rev. B: Condens. Matter Mater. Phys. 2006, 73, 165414.

(51) Klemm, H. W.; Peschel, G.; Madej, E.; Fuhrich, A.; Timma, M.; Menzel, D.; Schmidt, T.; Freund, H.-J. Preparation of Silica Films on $\mathrm{Ru}(0001)$ : A LEEM/PEEM Study. Surf. Sci. 2015, 3, 45-51.

(52) Lichtenstein, L.; Heyde, M.; Ulrich, S.; Nilius, N.; Freund, H.-J. Probing the properties of metal-oxide interfaces: silica films on Mo and Ru supports. J. Phys.: Condens. Matter 2012, 24, 354010.

(53) Mitchell, W. J.; Weinberg, W. H. Interaction of $\mathrm{NO}_{2}$ with $\mathrm{Ru}(001)$ : Formation and Decomposition of $\mathrm{RuO}_{\mathrm{x}}$ Layers. J. Chem. Phys. 1996, 104, 9127-9136.

(54) Böttcher, A.; Niehus, H. Formation of Subsurface Oxygen at $\mathrm{Ru}(0001)$. J. Chem. Phys. 1999, 110, 3186-3195. 